Published in final edited form as:

J Cardiovasc Comput Tomogr. 2019 ; 13(4): 196-202. doi:10.1016/j.jcct.2019.05.009.

\title{
Non-invasive fractional flow reserve derived from coronary computed tomography angiography in patients with acute chest pain: Subgroup analysis of the ROMICAT II trial
}

\author{
Maros Ferencik $^{a, b, c}$, Michael T. Lu ${ }^{b, c}$, Thomas Mayrhofer ${ }^{b, c, d}$, Stefan B. Puchner ${ }^{b, c, e}$, Ting \\ Liu $^{b, c, f}$, Pal Maurovich-Horvat 9 , Khristine Ghemigian ${ }^{b, c}$, Alexander Ivanov ${ }^{b, c}$, Elizabeth \\ Adami $^{\mathrm{b}, c}$, John T. Nagurney ${ }^{\mathrm{h}}$, Pamela K. Woodardi, Quynh A. Truong', James E. Udelson ${ }^{\mathrm{k}}$, \\ Udo Hoffmann ${ }^{b, c}$
}

aKnight Cardiovascular Institute, Oregon Health \& Science University, Portland bepartment of Radiology, Massachusetts General Hospital and Harvard Medical School, Boston, MA ${ }^{\circ}$ Cardiac MR PET CT Program, Massachusetts General Hospital and Harvard Medical School, Boston, MA dSchool of Business Studies, Stralsund University of Applied Sciences, Stralsund, Germany eDepartment of Biomedical Imaging and Image-Guided Therapy, Medical University of Vienna, Vienna, Austria fDepartment of Radiology, First Affiliated Hospital of China Medical University, Shenyang, China 9MTA-SE Cardiovascular Imaging Research Group, Heart and Vascular Centre, Semmelweis University, Budapest, Hungary hDepartment of Emergency Medicine, Massachusetts General Hospital and Harvard Medical School, Boston, MA 'Mallinckrodt Institute of Radiology, Washington University School of Medicine, St. Louis, MO iDepartment of Radiology, New York-Presbyterian Hospital and Weill Cornell Medicine, New York, NY kDivision of Cardiology and the Cardio-Vascular Center, Tufts Medical Center, Boston, MA

\section{Abstract \\ Background-Non-invasive fractional flow reserve $\left(\mathrm{FFR}_{\mathrm{CT}}\right)$ derived from coronary computed tomography angiography (CTA) permits hemodynamic evaluation of coronary stenosis and may improve efficiency of assessment in stable chest pain patients. We determined feasibility of FFR $\mathrm{CT}_{\mathrm{C}}$ in the population of acute chest pain patients and assessed the relationship of FFR $\mathrm{CT}_{\mathrm{C}}$ with outcomes of acute coronary syndrome (ACS) and revascularization and with plaque characteristics.}

\begin{abstract}
Methods-We included 68 patients (mean age $55.8 \pm 8.4$ years, $71 \%$ men) from the ROMICAT II trial who had $250 \%$ stenosis on coronary CTA or underwent additional non-invasive stress test. We evaluated coronary stenosis and high-risk plaque on coronary CTA.
\end{abstract}

Corresponding author: Maros Ferencik, MD, PhD, MCR, Knight Cardiovascular Institute, Oregon Health \& Science University, 3180 Sam Jackson Park Rd., Mail Code UHN62, Portland, OR 97239, Phone: 503-494-5263, Fax: 503-494-8550, ferencik@ ohsu.edu. Supplementary Data Two tables and one figure.

Publisher's Disclaimer: This is a PDF file of an unedited manuscript that has been accepted for publication. As a service to our customers we are providing this early version of the manuscript. The manuscript will undergo copyediting, typesetting, and review of the resulting proof before it is published in its final citable form. Please note that during the production process errors may be discovered which could affect the content, and all legal disclaimers that apply to the journal pertain. 
FFR $_{\mathrm{CT}}$ was measured in a core laboratory.

Results-We found correlation between anatomic severity of stenosis and FFR $\mathrm{CT}_{\mathrm{C}} \unlhd \mathbf{0}$.80 vs. FFR $_{\mathrm{CT}}>0.80$ (severe stenosis $84.8 \%$ vs. $15.2 \%$; moderate stenosis $33.3 \%$ vs. $66.7 \%$; mild stenosis $33.3 \%$ vs. $66.7 \%$ patients). Patients with severe stenosis had lower FFR $_{\mathrm{CT}}$ values (median 0.64 , $25^{\text {th }}-75^{\text {th }}$ percentile $0.50-0.75$ ) as compared to patients with moderate (median $0.84,25^{\text {th }}-75^{\text {th }}$ percentile, $\mathrm{p}<0.001$ ) or mild stenosis (median $0.86,25^{\text {th }}-75^{\text {th }}$ percentile $0.78-0.88, \mathrm{p}<0.001$ ). The relative risk of ACS and revascularization in patients with positive $\mathrm{FFR}_{\mathrm{CT}} \unlhd$ ).80 was 4.03 (95\% CI $1.56-10.36)$ and 3.50 (95\% CI 1.12-10.96), respectively. FFR ${ }_{C T} \unlhd 0.80$ was associated with the presence of high-risk plaque (odds ratio $3.91,95 \%$ CI 1.55-9.85, p=0.004) after adjustment for stenosis severity.

Conclusion-Abnormal $\mathrm{FFR}_{\mathrm{CT}}$ was associated with the presence of ACS, coronary revascularization, and high-risk plaque. $\mathrm{FFR}_{\mathrm{CT}}$ measurements correlated with anatomic severity of stenosis on coronary CTA and were feasible in population of patients with acute chest pain.

\section{Keywords}

coronary computed tomography angiography; non-invasive fractional flow reserve; stress test; acute coronary syndrome; risk stratification; non-invasive cardiac testing

\section{Introduction}

Coronary computed tomography angiography (CTA) has become a standard method for the evaluation of patients who present to the emergency department (ED) with symptoms suggestive of acute coronary syndrome (ACS). ${ }^{1-3}$ Coronary CTA permits rapid evaluation of patients with decreased time to diagnosis and discharge as compared to standard work-up with stress testing. ${ }^{1-3}$ The major strength of coronary CTA is its high negative predictive value for the exclusion of significant coronary stenosis and ACS. However, the positive predictive value, especially in patients with intermediate stenosis, is only moderate. In patients with suspected ACS in the ED, this may result in higher rates of invasive coronary angiography (ICA) and coronary revascularization among patients who undergo coronary CTA as compared to stress testing. ${ }^{1-3}$

Supplemental analyses of coronary CTA datasets, such as the assessment of coronary plaque, first-pass perfusion and resting wall motion abnormalities, have been proposed to improve specificity and positive predictive value of the test for stenosis and ACS. ${ }^{4-6}$ More recently, non-invasive fractional flow reserve calculation from coronary CTA $\left(\mathrm{FFR}_{\mathrm{CT}}\right)$ has been developed. Using computational fluid dynamics, $\mathrm{FFR}_{\mathrm{CT}}$ can provide accurate assessment of fractional flow reserve with standard coronary CTA acquisition and no need for adenosine infusion. The measurements of $\mathrm{FFR}_{\mathrm{CT}}$ correlated closely with invasive FFR measurements. ${ }^{7,8}$ The evaluation of hemodynamic significance is important as there is frequent discordance between anatomic severity of stenosis and its hemodynamic significance. ${ }^{9,10}$ Furthermore, coronary revascularization guided by the hemodynamic assessment with invasive FFR lead to improved outcomes as compared to the anatomic assessment only. ${ }^{11}$ Management strategy of coronary CTA with FFR $_{\mathrm{CT}}$ was demonstrated to be feasible and safe alternative of ICA, with overall lower rate of ICA and equivalent clinical 
outcomes with lower cost at 1-year followup. ${ }^{12,13} \mathrm{FFR}_{\mathrm{CT}}$ was also a better predictor of need for revascularization and future major adverse cardiovascular events when compared to anatomic stenosis detected by coronary CTA. ${ }^{14}$ There is growing evidence that abnormal $\mathrm{FFR}_{\mathrm{CT}}$ is not only related to anatomic severity of stenosis, but also to coronary plaque characteristics and burden. ${ }^{15-18}$

The majority of $\mathrm{FFR}_{\mathrm{CT}}$ studies has been performed in stable chest pain patients in the outpatient setting. The feasibility and performance of $\mathrm{FFR}_{\mathrm{CT}}$ in the acute chest pain setting has not been studied. Therefore, we analyzed data from the Rule Out Myocardial Infarction/ Ischemia Using Computer Assisted Tomography (ROMICAT) II trial and performed FFR $\mathrm{CT}_{\mathrm{C}}$ measurements in a subgroup of patients to determine the feasibility and performance of the technique in the population of acute chest pain patients in the ED. We also performed advanced coronary plaque analyses to assess the relationship between plaque characteristics and $\mathrm{FFR}_{\mathrm{CT}}$ and their relation to ACS outcomes.

\section{Methods}

\section{Patient population}

The study population was selected from patients who were enrolled in the ROMICAT II trial, were randomized to the coronary CTA arm of the trial and underwent coronary CTA. ${ }^{2}$ The ROMICAT II trial enrolled patients with acute chest pain and a low-to-intermediate suspicion for ACS. The local institutional review boards approved the study. In our retrospective study, we evaluated patients who had $250 \%$ stenosis as determined by the local site reads of coronary CTA or underwent an additional non-invasive cardiac stress test (Figure 1)

\section{Coronary CTA}

Coronary CTA images were acquired using either retrospectively ECG-gated or prospectively ECG-triggered protocols. The investigators in the study used the scanners from three vendors (Siemens, General Electric, Toshiba) and different scanner generations (64-, 128-, 256-row, and dual source). Local physicians interpreted coronary CTA images and the presence of stenosis was categorized as severe ( $250 \%$ stenosis in the left main coronary artery or $\geq 70 \%$ in any coronary artery) or moderate (50\%-70\% stenosis in any coronary artery). Coronary CTA was deemed indeterminate if the readers were unable to rule out the presence of moderate or severe coronary stenosis.

\section{Computation of $F F R_{\mathrm{CT}}$ from coronary CTA}

The datasets submitted for the $\mathrm{FFR}_{\mathrm{CT}}$ analysis met prospectively established inclusion criteria (field of view $<250 \mathrm{~mm}$, slice thickness $<1 \mathrm{~mm}$, administration of nitroglycerin). The $\mathrm{FFR}_{\mathrm{CT}}$ implementation employed in this study was performed at a single core laboratory (HeartFlow Inc., Redwood City, California). CTA datasets meeting eligibility criteria were sent to the $\mathrm{FFR}_{\mathrm{CT}}$ core laboratory. As previously validated, $\mathrm{FFR}_{\mathrm{CT}}$ core laboratory applied a second set of quantitative criteria to determine whether image quality was adequate for $\mathrm{FFR}_{\mathrm{CT}}$ analysis based on clear definition of coronary artery lumen and myocardial 
boundaries. ${ }^{8}$ CTA datasets with inadequate image quality due to motion artifacts, severe calcium blooming artifacts or excessive image noise were excluded from the analysis.

$\mathrm{FFR}_{\mathrm{CT}}$ was calculated blinded to all aspects of clinical care and clinical outcomes. The results of $\mathrm{FFR}_{\mathrm{CT}}$ were not available to care providers. The techniques for calculation of FFR $_{C T}$ have been detailed and accuracy against invasive FFR validated previously. ${ }^{7,8}$ Briefly, 3-dimensional models of the coronary arterial tree and myocardium were segmented from the standard CTA images. Computational fluid dynamics techniques modeled coronary arterial flow under simulated maximal hyperemia. $\mathrm{FFR}_{\mathrm{CT}}$ was calculated as the ratio of mean simulated pressure to aortic pressure at all coronary artery locations measuring $\geq 1.8$ $\mathrm{mm}$ in diameter. Occluded vessels were assigned a value of 0.5 . The lowest per-patient $\mathrm{FFR}_{\mathrm{CT}}$ value was reported; a FFR $\mathrm{CT} \quad \boldsymbol{\$} 0.80$ at any coronary location constituted a per-patient "positive" result. We also reported per-vessel lowest FFR $_{C T}$ value, which was used in pervessel analysis. A FFR $\mathrm{CT}_{\mathrm{C}} \mathbb{} \mathbf{0} .80$ at any coronary location in a given vessel constituted a pervessel "positive" result. Transfer of data, image segmentation, FFR $_{\mathrm{CT}}$ calculation and reporting typically requires less than 2 hours with the current iteration of the technology.

\section{Invasive and non-invasive tests}

Investigators in the ROMICAT II trial prospectively collected information on ICA, revascularization procedures (percutaneous coronary intervention or coronary artery by-pass grafting) and additional non-invasive stress tests. Non-invasive stress tests included exercise treadmill electrocardiograms, nuclear myocardial perfusion imaging or stress echocardiograms. The tests were evaluated by local physicians and were reported positive if there was evidence of stress-induced ischemia.

\section{Core laboratory assessment of coronary CTA}

We performed additional core laboratory assessment of coronary CTA datasets. Three independent readers with level III experience in coronary CTA who were blinded to clinical care results evaluated coronary CTA on a per coronary segment basis using the model of the Society of Cardiovascular Computed Tomography. ${ }^{19}$ Each segment was assessed for the presence of coronary stenosis and plaque. The severity of stenosis was categorized as: $0 \%=$ no stenosis, $1-49 \%=$ non-obstructive coronary artery disease (CAD), 50-69\% = moderate stenosis, $250 \%$ stenosis in the left main coronary artery or $\geq 70 \%$ in any coronary artery $=$ severe stenosis.

Qualitative assessment for high-risk coronary plaque was performed in all coronary segments with plaque. High-risk plaque was defined as the presence of at least one of the following features: positive remodeling (remodeling index $>1.1$ ), presence of plaque with low $\mathrm{CT}$ attenuation of $<30 \mathrm{HU}$, napkin-ring sign and spotty calcium as described previously. 4

Four independent readers with level III experience in coronary CTA performed quantitative coronary CTA analysis in coronary segments with visually detectable plaques. The images were analyzed on a dedicated workstation (QAngio CT RE 2.0, Medis, Leiden, the Netherlands). Readers performed automatic detection of the coronary arteries and segmentation of luminal and outer vessel boundaries followed by manual adjustments of the 
vessel centerline and boundaries as needed. Reader then determined the proximal and distal reference of the coronary plaque in the adjacent normal vessel. The final results of the coronary CTA analysis were reported per coronary artery. We measured total plaque volume, volume of plaque with $<30 \mathrm{HU}$ and maximum remodeling index. The detailed description of the quantitative analysis was reported previously. ${ }^{20}$

\section{Acute coronary syndrome definition}

An independent clinical events committee adjudicated the presence of ACS during the index hospitalization as described previously. ${ }^{2}$ ACS was defined as acute myocardial infarction or unstable angina pectoris according to the American College of Cardiology/American Heart Association Guidelines. $^{21}$

\section{Statistical methods}

All statistical analyses were performed using Stata 13.1 (StataCorp LP, College Station, Texas). Continuous data are presented as mean \pm standard deviation or median and $25^{\text {th }}-75^{\text {th }}$ percentile. Categorical and ordinal variables are presented as absolute and relative frequencies. Comparisons for unpaired data were performed with the use of an independent sample $t$-test or the Wilcoxon rank-sum test for continuous variables, the Fisher exact test for categorical variables, and the Wilcoxon rank-sum test for ordinal variables. Binominal 95\% confidence intervals (CI) were calculated using the 'exact' method, i.e. ClopperPearson intervals. ${ }^{22}$ We performed multivariable multilevel mixed-effects logistic regression to evaluate the association between high-risk plaque (defined as any high-risk plaque by qualitative analysis) and $\mathrm{FFR}_{\mathrm{CT}}$ after adjustment for stenosis severity at vessel level. At patient level, we used multivariable logistic regression models, in which we studied the association of high-risk plaque, $\mathrm{FFR}_{\mathrm{CT}}$ and severe stenosis with the outcome of ACS during the index hospitalization. Based on these logistic regression models we calculated receiver operating characteristic (ROC) curves and corresponding areas under the receiver operating characteristics curve (AUC), which were compared using the DeLong algorithm. ${ }^{23}$ For all analyses, a 2-tailed $P$ value $<0.05$ was required to reject the null hypothesis.

\section{Results}

\section{Study population}

Of the 501 patients randomized to coronary CTA, 473 patients underwent and completed CTA scan (Figure 1). We included patients with $250 \%$ stenosis in at least one coronary artery $(n=82,17.3 \%)$ based on the site reads of coronary CTA. We also included patients who underwent an additional non-invasive cardiac test $(n=34,7.2 \%)$, among them 27 patients who had stenosis $<50 \%$ and 7 patients who had indeterminate stenosis severity. Total of 116 patients met inclusion criteria and their coronary CTA images were submitted for $\mathrm{FFR}_{\mathrm{CT}}$ analysis. Out of 116 patients, $48(41.4 \%)$ coronary CTA datasets were inadequate for $\mathrm{FFR}_{\mathrm{CT}}$ analysis due to motion artifacts, severe calcium blooming artifacts or excessive image noise. The characteristics of 68 subjects included in the study stratified by FFR $\mathrm{CT}_{\mathrm{T}}$ results are summarized in Table 1. There were no significant differences in baseline demographics and cardiovascular risk factors between subjects with $\mathrm{FFR}_{\mathrm{CT}} \unlhd \mathbf{} \mathrm{g} .80$ and $\mathrm{FFR}_{\mathrm{CT}}>0.80$ except for more men among those with abnormal FFR ${ }_{\mathrm{CT}} \$ \mathbf{0} .80$. Compared to 
those patients in whom $\mathrm{FFR}_{\mathrm{CT}}$ calculation was not feasible, there were no significant differences in baseline characteristics of patients with available FFR $_{\mathrm{CT}}$ measurements (Table S1).

\section{Coronary CTA findings and FFR}

We observed association between abnormal FFR $\mathrm{CT}_{\mathrm{C}} \boldsymbol{\$} \mathbf{0 . 8 0}$ and the presence of severe stenosis defined as stenosis $\geq 70 \%$ in any artery or $250 \%$ stenosis in the left main coronary artery as assessed by site reads of coronary CTA (Table 2). Among 33 patients with severe stenosis on coronary CTA, $28(84.8 \%)$ had FFR ${ }_{\mathrm{CT}} \unlhd 0.80$ and $5(15.2 \%)$ had FFR $\mathrm{CT}>0.80$ (Figure 2). Mild stenosis $(<50 \%)$ or moderate stenosis $(50-69 \%)$ was more often found in patients with normal FFR $\mathrm{CT}_{\mathrm{CT}}>0.80$ (Table 2). Patients with severe stenosis had lower FFR $\mathrm{CT}_{\mathrm{C}}$ values (median $0.64,25^{\text {th }}-75^{\text {th }}$ percentile $0.50-0.75$ ) as compared to patients with moderate (median $0.84,25^{\text {th }}-75^{\text {th }}$ percentile $0.75-0.90, \mathrm{p}<0.001$ ) or mild stenosis (median 0.86 , $25^{\text {th }}-75^{\text {th }}$ percentile $0.78-0.88, p<0.001$ ) (Figure 3 ).

\section{Association of FFR $\mathrm{RT}_{\mathrm{CT}}$ with ACS and coronary revascularization}

ACS during the index hospitalization was diagnosed in $39.7 \%$ (27/68) patients (myocardial infarction $n=3$, unstable angina pectoris $n=24$ ). ACS was more often diagnosed in patients with $\mathrm{FFR}_{\mathrm{CT}} \unlhd \mathbf{} \mathbf{0} .80\left(2 \frac{3}{3} 40\right.$ patients, $\left.57.5 \%\right)$ as compared to those with $\mathrm{FFR}_{\mathrm{CT}}>0.8(4 / 28$ patients, 14.3\%; $\mathrm{p}<0.001$, Table 2). Subjects with $\left.\mathrm{FFR}_{\mathrm{CT}} ₫\right) .80$ also underwent revascularization during the index hospitalization more often: 15/40 (37.5\%) patients vs. $3 / 28(10.7 \%)$ patients $(\mathrm{p}=0.024)$. The relative risk of ACS and revascularization in those with $\mathrm{FFR}_{\mathrm{CT}} \unlhd \mathbf{} \mathbf{0} .80$ as compared to those with $\mathrm{FFR}_{\mathrm{CT}}>0.8$ was 4.03 (95\% CI 1.56-10.36) and 3.50 (95\% CI 1.12-10.96), respectively.

\section{Potential effects of FFR $\mathrm{R}_{\mathrm{CT}}$ on downstream testing}

ICA was performed in $44.1 \%$ (30/68) patients (Table 2). Among them, $73.3 \%(22 / 30)$ had severe stenosis on coronary CTA. Moderate stenosis was found in $20.0 \%(6 / 30)$ and mild stenosis in $6.7 \%(2 / 30)$ patients on coronary CTA. In patients with $\mathrm{FFR}_{\mathrm{CT}}>0.8$ who underwent ICA, 37.5\% (3/8) patients had severe stenosis on ICA. Among 8 patients with moderate or mild stenosis on coronary CTA, 7 patients had normal $\mathrm{FFR}_{\mathrm{CT}}>0.8$ and only one patient with normal $\mathrm{FFR}_{\mathrm{CT}}$ was diagnosed with ACS.

Additional non-invasive stress test after coronary CTA was performed in $47.1 \%(32 / 68)$ patients. Among these patients, ACS was diagnosed in $18.8 \%$ (6/32). Abnormal FFR $_{\mathrm{CT}}$ $₫ 0.80$ was detected in $46.9 \%(15 / 32)$ patients. In the group of patients with $F_{F R} R_{C T} \unlhd 0.80$, $26.7 \%$ (4/15) patients were diagnosed with ACS. Normal FFR $\mathrm{CT}>0.80$ was found in $53.1 \%$ (17/32) patients. The result of non-invasive stress test was positive for ischemia in $17.7 \%$ (3/17) patients with $\mathrm{FFR}_{\mathrm{CT}}>0.80$. Two patients from this group were diagnosed with ACS $\left(11.8 \%, 2 / 17, \mathrm{p}=0.383\right.$ as compared to abnormal $\left.\mathrm{FFR}_{\mathrm{CT}}\right)$. Coronary CTA showed severe stenosis in $5.9 \%$ (1/17) patients. The remaining patients had moderate $(29.4 \%, 5 / 17)$, mild $(58.8 \%, 10 / 17)$ or indeterminate $(5.9 \%, 1 / 17)$ stenosis. Only one patient without severe stenosis on coronary CTA and $\mathrm{FFR}_{\mathrm{CT}}>0.8$ was diagnosed with ACS. FFR $\mathrm{CT}_{\mathrm{T}}$ could potentially decrease the need for additional non-invasive testing in half of patients $(16 / 32$ with less than severe stenosis on coronary CTA and normal FFR $_{\mathrm{CT}}$ ). 


\section{Core laboratory evaluation of high-risk plaque and $F F R_{C T}$}

We performed additional analysis with core laboratory assessment of coronary CTA. Among 16 patients with severe stenosis on coronary CTA, all 16 (100\%) patients had positive

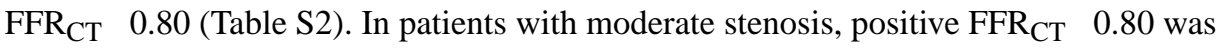

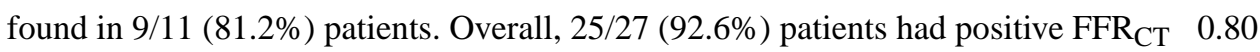
among patients with moderate or severe stenosis by core laboratory reads. In contrast, only $34 / 51(66.7 \%)$ patients with moderate or severe stenosis by site reads had positive FFR $_{\mathrm{CT}}$ $\$$ \$.80. Among patients with mild stenosis by core laboratory reads, $26 / 41(63.4 \%)$ had negative $\mathrm{FFR}_{\mathrm{CT}}>0.80$, which was similar to site reads (10/15 patients, $\left.66.7 \%\right)$.

In a per vessel analysis, we found good correlation between coronary stenosis and $\mathrm{FFR}_{\mathrm{CT}}$ as assessed by the core laboratory (Table 3). Vessels with abnormal FFR $\mathrm{CT}_{\mathbf{S}} ₫ 0.8$ had higher prevalence of high-risk plaque (any high-risk plaque and individual high-risk plaque features of positive remodeling, low CT attenuation plaque, napkin-ring sign and spotty calcium) as detected by qualitative analysis. Similar results were found in per patient analysis (data not shown). In quantitative plaque analysis, we observed higher remodeling index, total plaque volume and low $\mathrm{CT}$ attenuation ( $<30 \mathrm{HU}$ ) plaque volume in vessels with abnormal $\mathrm{FFR}_{\mathrm{CT}}$ $₫$ 0.8. In multivariable multilevel mixed-effects logistic regression, the association between high-risk plaque (defined as any high-risk plaque by qualitative analysis) and FFR $_{\mathrm{CT}} \unlhd \mathbf{} \mathbf{} .8$ was independent of stenosis severity (odds ratio $3.91,95 \%$ CI $1.55-9.85, \mathrm{p}=0.004$ ). In the multivariable model at patient level, the addition of high-risk plaque (odds ratio 16.47, 95\%CI 1.13-240.81, $\mathrm{p}=0.041$ ) and $\mathrm{FFR}_{\mathrm{CT}}$ (odds ratio 1.67, 95\% CI 0.40-7.09, $\mathrm{p}=0.483$ ) to stenosis (odds ratio 40.18, 95\%CI 3.44-469.36, $\mathrm{p}=0.003$ ) improved discriminatory capacity for ACS as compared to stenosis only (AUC: $0.87,95 \%$ CI $0.79-0.95$ vs. 0.77 , $95 \%$ CI $0.67-$ $0.86 ; \mathrm{p}=0.003$; Figure $\mathrm{S} 1$ ).

\section{Discussion}

We performed analysis of non-invasive $\mathrm{FFR}_{\mathrm{CT}}$ in patients from the ROMICAT II trial who underwent coronary CTA and had moderate or severe coronary stenosis or required additional non-invasive stress test. We demonstrated the feasibility of $\mathrm{FFR}_{\mathrm{CT}}$ analysis in acute chest pain population in multicenter, multivendor setting. Our study showed that severity of stenosis on coronary CTA correlated with $\mathrm{FFR}_{\mathrm{CT}}$ measurements. Patients with abnormal $\mathrm{FFR}_{\mathrm{CT}}$ were also more likely diagnosed with ACS and more often required revascularization. In coronary plaque analysis, we observed association of the presence of high-risk plaque features with abnormal FFR $_{\mathrm{CT}}$ values, which persisted after adjustment for stenosis severity.

\section{Feasibility of $F F R_{C T}$ in acute chest pain population}

Prior studies reported good correlation of $\mathrm{FFR}_{\mathrm{CT}}$ with invasive FFR as well as relationship to outcomes in populations of outpatients with stable chest pain. ${ }^{7,8,12-14,24}$ Large multicenter trials reported that $71-89 \%$ of coronary CTA datasets were of sufficient images quality for $\mathrm{FFR}_{\mathrm{CT}}$ analysis. ${ }^{7,8,13}$ The results from clinical practice with focused image optimization required for $\mathrm{FFR}_{\mathrm{CT}}$ showed event better results with 97-99\% of coronary CTA having image quality sufficient for $\mathrm{FFR}_{\mathrm{CT}}$ analysis. ${ }^{25,26}$ In our study, we demonstrated that 
the evaluation of $\mathrm{FFR}_{\mathrm{CT}}$ in patients with acute chest pain in the ED was feasible. We selected a population with high prevalence of $\mathrm{CAD}$ and patients who required additional testing. Despite the challenging population characteristics, we observed that $\mathrm{FFR}_{\mathrm{CT}}$ was feasible in 59\% of patients. This was possible despite the fact that coronary CTA scans were not acquired with the goal to perform $\mathrm{FFR}_{\mathrm{CT}}$ and were acquired before 2011, i.e. with older $\mathrm{CT}$ equipment. The $\mathrm{FFR}_{\mathrm{CT}}$ acceptance was similar to the analysis from the PROspective Multicenter Imaging Study for Evaluation of Chest Pain (PROMISE), in which 67\% of coronary CTA datasets were evaluable. ${ }^{14}$ In the future prospective studies of $\mathrm{FFR}_{\mathrm{CT}}$ in acute chest pain populations, the investigators will have to pay attention to obtain high image quality necessary for the analysis. Rapid evaluation in the core laboratory with turnaround time of a few hours will be necessary for the implementation of $\mathrm{FFR}_{\mathrm{CT}}$ into the ED workflow. In addition to $\mathrm{FFR}_{\mathrm{CT}}$ performed as an offsite analysis, which may require longer processing times, on-site $\mathrm{FFR}_{\mathrm{CT}}$ solutions tested in smaller, single center studies have been demonstrated to provide results that compare favorably with invasive FFR and have been feasible in patients with suspected ACS. ${ }^{17,24,25}$ Considering on-site assessment may be more time efficient, especially in the setting of acute chest pain where efficient time management is crucial, but future studies will be needed to evaluate this concept.

\section{Correlation of FFR $\mathbf{R T}_{\mathrm{CT}}$ with stenosis and ACS outcomes}

Prior studies showed correlation of $\mathrm{FFR}_{\mathrm{CT}}$ with stenosis severity ${ }^{14}$, which was confirmed in our study. However, there was still substantial disagreement. Mild or moderate stenosis was found on coronary CTA in $28 \%$ (11/40) patients with abnormal FFR $\mathrm{CT}_{\mathrm{CT}} \mathbf{\Delta} .8$. There were $18 \%$ $(5 / 28)$ patients with severe stenosis as assessed by site coronary CTA reads who had normal $\mathrm{FFR}_{\mathrm{CT}}>0.8$. Therefore, overall there was disagreement in $24 \%(16 / 68)$ patients. These findings are similar to the results from the PROMISE trial, in which there was $31 \%$ of patients with disagreement between coronary CTA and FFR $\mathrm{CT}_{\mathrm{T}}$ and $29 \%$ of patients with disagreement between ICA and FFR $\mathrm{CT}^{.}{ }^{14}$ The disagreement was higher in the Analysis of Coronary Blood Flow Using CT Angiography: Next Steps (NXT) trial, in which the investigators observed disagreement between coronary CTA and $\mathrm{FFR}_{\mathrm{CT}}$ in $47 \%$ of patients. ${ }^{8}$ Our results are also in line with experience comparing invasive evaluation of ICA with FFR, which showed discrepancy rate of $25 \%$ in the Fractional Flow Reserve Versus Angiography in Multivessel Evaluation (FAME) trial. ${ }^{10}$

An interesting observation from our study is the difference in the agreement between coronary CTA and $\mathrm{FFR}_{\mathrm{CT}}$ when core laboratory evaluation was used. We found that all patients graded as having severe stenosis on coronary CTA by core laboratory readers were also found to have $\mathrm{FFR}_{\mathrm{CT}} \mathfrak{\$} \mathbf{0 . 8}$. Overall, we found lower grading of stenosis severity in core laboratory reads. The results are in concordance with the recent analysis from the PROMISE trial that showed lower prevalence of significant stenosis in core laboratory CTA reads as compared to the site reads. ${ }^{26}$ Less conservative assessment of stenosis severity (i.e. less patients with significant stenosis) did not affect the predictive accuracy of CTA for the future majors adverse cardiovascular events. ${ }^{26}$ These results suggest that combined "less conservative" evaluation of coronary CTA for stenosis with detection of hemodynamically significant lesions using $\mathrm{FFR}_{\mathrm{CT}}$ is a concept that will need to be prospectively evaluated. 
We evaluated the association of $\mathrm{FFR}_{\mathrm{CT}}$, which was not used for the clinical decision-making in the ROMICAT II trial, with the outcome of ACS during the index hospitalization. There was a strong association between abnormal $\mathrm{FFR}_{\mathrm{CT}}$ and the diagnosis of ACS. Among 27 patients with the diagnosis of ACS, 23 (85\%) patients had FFR $\left.\mathrm{CT}_{\mathbf{S}} \mathbf{\$}\right) .8$. This observation showed that hemodynamic significance of coronary lesions is important predictor of ACS in acute chest pain setting. Our results mirror the prior experience from stable chest pain population. In the PROMISE trial, the association of $\mathrm{FFR}_{\mathrm{CT}}$ with revascularization and major adverse cardiovascular events was significantly stronger than for severe stenosis on CTA (hazard ratio 4.31 vs. $2.90, \mathrm{p}=0.033$ ), a notable result as stenosis guided the clinical decision for coronary revascularization, whereas $\mathrm{FFR}_{\mathrm{CT}}$ was not available to caregivers in the trial. ${ }^{14}$

Four out of 28 patients with $\mathrm{FFR}_{\mathrm{CT}}>0.8$ were diagnosed with ACS. These discrepant results, reflect clinical practice, in which further testing may be driven by clinical presentation in acute chest pain. Furthermore, prior studies showed remaining discordance of $\mathrm{FFR}_{\mathrm{CT}}$ results with invasive FFR, especially around the threshold of 0.8 , and between FFR $_{\mathrm{CT}}$ and functional stress testing. ${ }^{27,28}$

\section{FFR $_{\mathrm{CT}}$ and coronary plaque characteristics}

The presence of high-risk plaque features, such as positive remodeling, low CT attenuation, napkin-ring sign and spotty calcium, on coronary CTA is associated with the presence of ACS in acute setting and increased risk of future cardiovascular events in stable chest pain patients. ${ }^{4,29}$ In a secondary analysis of Determination of Fractional Flow Reserve by Anatomic Computed Tomographic Angiography (DeFACTO) trial of stable chest pain patients, qualitative and quantitative measures of coronary plaque were independently associated with lesions causing ischemia as confirmed by invasive FFR. ${ }^{16}$ Similar observations were made by the investigators in the NXT trial who found association of significant stenosis, non-calcified and low $\mathrm{CT}$ attenuation plaque volume with abnormal FFR $_{\mathrm{CT}} \unlhd 3.8 .^{15}$ The volume of low CT attenuation plaque and FFR $\mathrm{CT}_{\text {T }}$ was incremental to stenosis in prediction of lesion specific ischemia as assessed by invasive FFR. The incremental value of plaque assessment and $\mathrm{FFR}_{\mathrm{CT}}$ was also present in patients with nonsignificant stenosis on coronary CTA. We extended this novel observation of the association between high-risk plaque features and total and low $\mathrm{CT}$ attenuation plaque volume with hemodynamic significance as assessed by $\mathrm{FFR}_{\mathrm{CT}}$, which was independent of the presence of significant stenosis, in the acute chest pain population. Our results provide an additional piece of evidence supporting the notion that coronary plaque characteristics are an important determinant of hemodynamic significance of coronary stenosis.

\section{Limitations}

We performed FFR $_{\mathrm{CT}}$ analysis in a subgroup of patients in the ROMICAT II trial. This approach may introduce selection bias. The results of coronary CTA based on site reads affected the clinical decisions and were also used to select the patients included in our substudy. Only 3 out of 22 patients diagnosed with ACS had myocardial infarction. Remaining 19 patients were diagnosed with unstable angina. This limits our ability to study relationship between stenosis detected by site reads and $\mathrm{FFR}_{\mathrm{CT}}$ with respect to the 
prediction of ACS. The estimates of decreased need for secondary testing and ICA are affected by those limitations as well. Further prospective studied will be necessary to evaluate whether implementation of $\mathrm{FFR}_{\mathrm{CT}}$ in the evaluation of patients with acute chest pain in the ED can decrease the rates of secondary testing. Invasive FFR was not performed routinely in the ROMICAT II trial. Therefore, we were unable to confirm the results of FFR $_{C T}$ with invasive FFR in patients who underwent ICA. A fairly high proportion of coronary CTAs were not suitable for $\mathrm{FFR}_{\mathrm{CT}}$ analysis, which is reflective of older generations of CT scanners and image acquisition not being optimized for $\mathrm{FFR}_{\mathrm{CT}}$.

\section{Conclusions}

We showed that $\mathrm{FFR}_{\mathrm{CT}}$ measurements correlated with the anatomic severity of stenosis on coronary CTA. Abnormal FFR $\mathrm{CT}_{\mathrm{T}}$ was associated with the presence of ACS and coronary revascularization. We found association between the presence of high-risk plaque and $\mathrm{FFR}_{\mathrm{CT}}$, which was independent of stenosis severity.

\section{Supplementary Material}

Refer to Web version on PubMed Central for supplementary material.

\section{Acknowledgements}

The authors thank Drs. Campbell Rogers and Souma Sengupta (Heart Flow) for calculation of FFRCT.

Disclosures

ROMICAT II Support NIH U01HL092040 and U01HL092022, ACRIN

Ferencik: Research Grant/Significant: American Heart Association Fellow to Faculty Award 13FTF16450001

Lu: Research Grant/Significant: American Roentgen Ray Society Scholarship.

Truong: Research Grant/Significant: NIH/NHLBI K23HL098370 and L30HL093896, St. Jude Medical, American College of Radiology Imaging Network, and Duke Clinical Research

Nagurney: Research Grant/Significant: Alere/Biosite, Brahms Ltd/Thermo-Fisher, Nanosphere; Consultant/ Advisory/Significant: Board CardioDx

Hoffmann: Research Grant/Significant: NIH U01HL092040, U01HL092022, Siemens Medical Solutions, Heart Flow Inc; Consultant/Advisory Board/Significant: Heart Flow All other authors have reported that they have no relationships relevant to the contents of this paper to disclose.

\section{References}

1. Goldstein JA, Chinnaiyan KM, Abidov A, et al. The CT-STAT (Coronary Computed Tomographic Angiography for Systematic Triage of Acute Chest Pain Patients to Treatment) Trial. J Am Coll Cardiol. 2011;58(14):1414-1422. doi:10.1016/j.jacc.2011.03.068. [PubMed: 21939822]

2. Hoffmann U, Truong QA, Schoenfeld DA, et al. Coronary CT angiography versus standard evaluation in acute chest pain. N Engl J Med. 2012;367(4):299-308. doi:10.1056/NEJMoa1201161. [PubMed: 22830462]

3. Litt HI, Gatsonis C, Snyder B, et al. CT angiography for safe discharge of patients with possible acute coronary syndromes. N Engl J Med. 2012;366(15):1393-1403. doi:10.1056/NEJMoa1201163. [PubMed: 22449295]

4. Puchner SB, Liu T, Mayrhofer T, et al. High-risk plaque detected on coronary CT angiography predicts acute coronary syndromes independent of significant stenosis in acute chest pain: results 
from the ROMICAT-II trial. J Am Coll Cardiol. 2014;64(7):684-692. doi:10.1016/j.jacc. 2014.05.039. [PubMed: 25125300]

5. Seneviratne SK, Truong QA, Bamberg F, et al. Incremental diagnostic value of regional left ventricular function over coronary assessment by cardiac computed tomography for the detection of acute coronary syndrome in patients with acute chest pain: from the ROMICAT trial. Circ Cardiovasc Imaging. 2010;3(4):375-383. doi:10.1161/CIRCIMAGING.109.892638. [PubMed: 20484542]

6. Feuchtner GM, Plank F, Pena C, et al. Evaluation of myocardial CT perfusion in patients presenting with acute chest pain to the emergency department: comparison with SPECT-myocardial perfusion imaging. Heart. 2012;98(20):1510-1517. doi:10.1136/heartjnl-2012-302531. [PubMed: 22895647]

7. Min JK, Leipsic J, Pencina MJ, et al. Diagnostic accuracy of fractional flow reserve from anatomic CT angiography. JAMA. 2012;308(12):1237-1245. doi:10.1001/2012.jama.11274. [PubMed: 22922562]

8. Nørgaard BL, Leipsic J, Gaur S, et al. Diagnostic performance of noninvasive fractional flow reserve derived from coronary computed tomography angiography in suspected coronary artery disease: the NXT trial (Analysis of Coronary Blood Flow Using CT Angiography: Next Steps). J Am Coll Cardiol. 2014;63(12):1145-1155. doi:10.1016/j.jacc.2013.11.043. [PubMed: 24486266]

9. Budoff MJ, Nakazato R, Mancini GBJ, et al. CT Angiography for the Prediction of Hemodynamic Significance in Intermediate and Severe Lesions: Head-to-Head Comparison With Quantitative Coronary Angiography Using Fractional Flow Reserve as the Reference Standard. JACC Cardiovasc Imaging. 2016;9(5):559-564. doi:10.1016/j.jcmg.2015.08.021. [PubMed: 26897669]

10. Tonino PAL, Fearon WF, de Bruyne B, et al. Angiographic versus functional severity of coronary artery stenoses in the FAME study fractional flow reserve versus angiography in multivessel evaluation. J Am Coll Cardiol. 2010;55(25):2816-2821. doi:10.1016/j.jacc.2009.11.096. [PubMed: 20579537]

11. de Bruyne B, Pijls NHJ, Kalesan B, et al. Fractional flow reserve-guided PCI versus medical therapy in stable coronary disease. N Engl J Med. 2012;367(11):991-1001. doi:10.1056/ NEJMoa1205361. [PubMed: 22924638]

12. Douglas PS, de Bruyne B, Pontone G, et al. 1-Year Outcomes of FFRCT-Guided Care in Patients With Suspected Coronary Disease: The PLATFORM Study. J Am Coll Cardiol. 2016;68(5):435445. doi:10.1016/j.jacc.2016.05.057. [PubMed: 27470449]

13. Douglas PS, Pontone G, Hlatky MA, et al. Clinical outcomes of fractional flow reserve by computed tomographic angiography-guided diagnostic strategies vs. usual care in patients with suspected coronary artery disease: the prospective longitudinal trial of FFR(CT): outcome and resource impacts study. Eur Heart J. 2015;36(47):3359-3367. doi:10.1093/eurheartj/ehv444. [PubMed: 26330417]

14. Lu MT, Ferencik M, Roberts RS, et al. Noninvasive FFR Derived From Coronary CT Angiography: Management and Outcomes in the PROMISE Trial. JACC Cardiovasc Imaging. 2017;10(11):1350-1358. doi:10.1016/j.jcmg.2016.11.024. [PubMed: 28412436]

15. Gaur S, Øvrehus KA, Dey D, et al. Coronary plaque quantification and fractional flow reserve by coronary computed tomography angiography identify ischaemia-causing lesions. Eur Heart J. 2016;37(15):1220-1227. doi:10.1093/eurheartj/ehv690. [PubMed: 26763790]

16. Park H-B, Heo R, ó Hartaigh B, et al. Atherosclerotic plaque characteristics by CT angiography identify coronary lesions that cause ischemia: a direct comparison to fractional flow reserve. JACC Cardiovasc Imaging. 2015;8(1):1-10. doi:10.1016/j.jcmg.2014.11.002. [PubMed: 25592691]

17. Tesche C, De Cecco CN, Caruso D, et al. Coronary CT angiography derived morphological and functional quantitative plaque markers correlated with invasive fractional flow reserve for detecting hemodynamically significant stenosis. J Cardiovasc Comput Tomogr. 2016;10(3):199_ 206. doi:10.1016/j.jcct.2016.03.002. [PubMed: 26993434]

18. Driessen RS, Stuijfzand WJ, Raijmakers PG, et al. Effect of Plaque Burden and Morphology on Myocardial Blood Flow and Fractional Flow Reserve. J Am Coll Cardiol. 2018;71(5):499-509. doi:10.1016/j.jacc.2017.11.054. [PubMed: 29406855]

19. Leipsic J, Abbara S, Achenbach S, et al. SCCT guidelines for the interpretation and reporting of coronary CT angiography: a report of the Society of Cardiovascular Computed Tomography 
Guidelines Committee. J Cardiovasc Comput Tomogr. 2014;8(5):342-358. doi:10.1016/j.jcct. 2014.07.003. [PubMed: 25301040]

20. Ferencik M, Mayrhofer T, Puchner SB, et al. Computed tomography-based high-risk coronary plaque score to predict acute coronary syndrome among patients with acute chest pain--Results from the ROMICAT II trial. J Cardiovasc Comput Tomogr. 2015;9(6):538-545. doi:10.1016/j.jcct. 2015.07.003. [PubMed: 26229036]

21. Anderson JL, Adams CD, Antman EM, et al. 2011 ACCF/AHA Focused Update Incorporated Into the ACC/AHA 2007 Guidelines for the Management of Patients With Unstable Angina/Non-STElevation Myocardial Infarction: A Report of the American College of Cardiology Foundation/ American Heart Association Task Force on Practice Guidelines. Circulation. 2011;123(18):e426e579. doi:10.1161/CIR.0b013e318212bb8b. [PubMed: 21444888]

22. Clopper CJ, Pearson ES. The Use of Confidence or Fiducial Limits Illustrated in the Case of the Binomial. Biometrika. 1934;26(4):404-413. doi:10.2307/2331986.

23. Delong ER, Delong DM, Clarke-Pearson DL. Comparing the areas under two or more correlated receiver operating characteristic curves: a nonparametric approach. Biometrics. 1988;44(3):837_ 845. [PubMed: 3203132]

24. Ko BS, Cameron JD, Munnur RK, et al. Noninvasive CT-Derived FFR Based on Structural and Fluid Analysis: A Comparison With Invasive FFR for Detection of Functionally Significant Stenosis. JACC Cardiovasc Imaging. 2017;10(6):663-673. doi:10.1016/j.jcmg.2016.07.005. [PubMed: 27771399]

25. Duguay TM, Tesche C, Vliegenthart R, et al. Coronary Computed Tomographic AngiographyDerived Fractional Flow Reserve Based on Machine Learning for Risk Stratification of NonCulprit Coronary Narrowings in Patients with Acute Coronary Syndrome. Am J Cardiol. 2017;120(8):1260-1266. doi:10.1016/j.amjcard.2017.07.008. [PubMed: 28844517]

26. Lu MT, Meyersohn NM, Mayrhofer T, et al. Central Core Laboratory versus Site Interpretation of Coronary CT Angiography: Agreement and Association with Cardiovascular Events in the PROMISE Trial. Radiology. 2018;287(1):87-95. doi:10.1148/radiol.2017172181. [PubMed: 29178815]

27. Cook CM, Petraco R, Shun-Shin MJ, et al. Diagnostic Accuracy of Computed TomographyDerived Fractional Flow Reserve : A Systematic Review. JAMA Cardiol. 2017;2(7):803-810. doi: 10.1001/jamacardio.2017.1314. [PubMed: 28538960]

28. Driessen RS, Danad I, Stuijfzand WJ, et al. Comparison of Coronary Computed Tomography Angiography, Fractional Flow Reserve, and Perfusion Imaging for Ischemia Diagnosis. J Am Coll Cardiol. 2019;73(2):161-173. doi:10.1016/j.jacc.2018.10.056. [PubMed: 30654888]

29. Motoyama S, Ito H, Sarai M, et al. Plaque Characterization by Coronary Computed Tomography Angiography and the Likelihood of Acute Coronary Events in Mid-Term Follow-Up. J Am Coll Cardiol. 2015;66(4):337-346. doi:10.1016/j.jacc.2015.05.069. [PubMed: 26205589] 
Patients randomized to coronary CTA arm of the ROMICAT II trial $n=501$

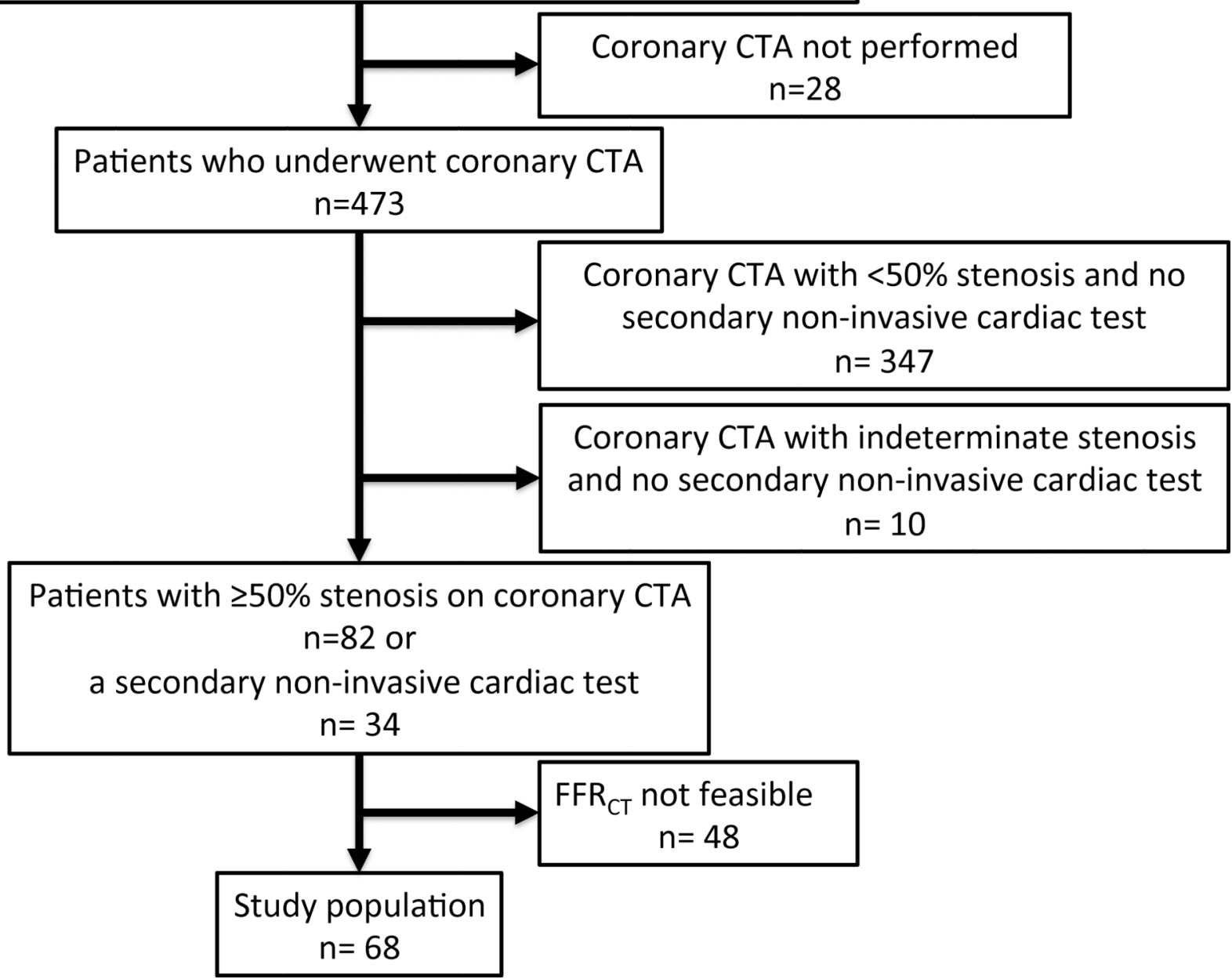

Figure 1.

Patient inclusions and exclusions. 

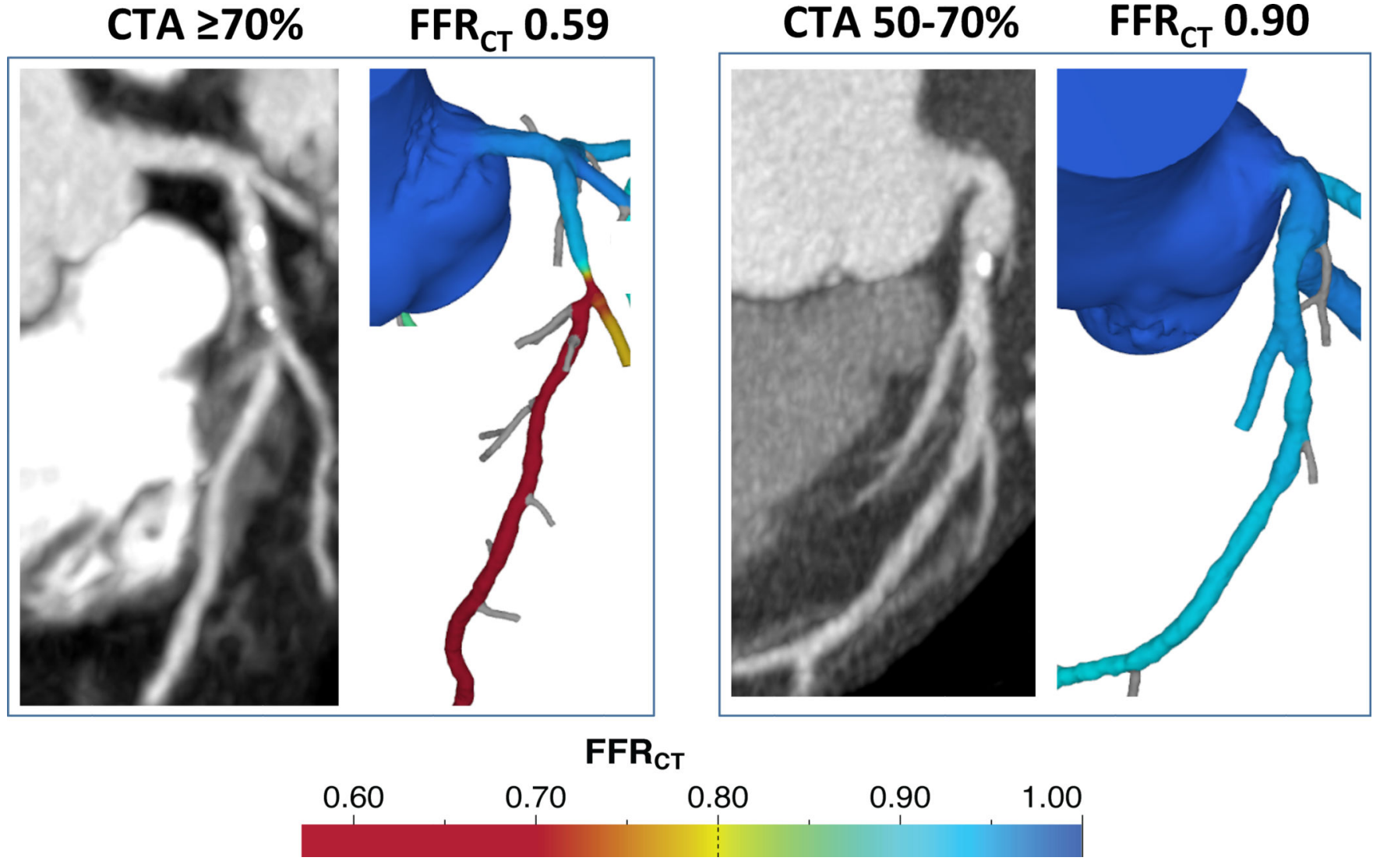

Figure 2.

Representative cases of coronary CTA and FFR $\mathrm{CT}_{\mathrm{C}}$. Left panel: 64-year-old man with acute chest pain diagnosed with unstable angina. Coronary CTA demonstrated 70-99\% stenosis of the left anterior descending coronary artery at the origin of the first diagonal branch. FFR ${ }_{\mathrm{CT}}$ was abnormal (0.59). Invasive coronary angiography confirmed $\geq 70 \%$ stenosis and patient underwent stent placement. Right panel: 61-year-old woman diagnosed with non-cardiac chest pain. Coronary CTA demonstrated 50-70\% stenosis in mid left anterior descending coronary artery. $\mathrm{FFR}_{\mathrm{CT}}$ was normal (0.90). Invasive coronary angiography showed 30-50\% stenosis. 


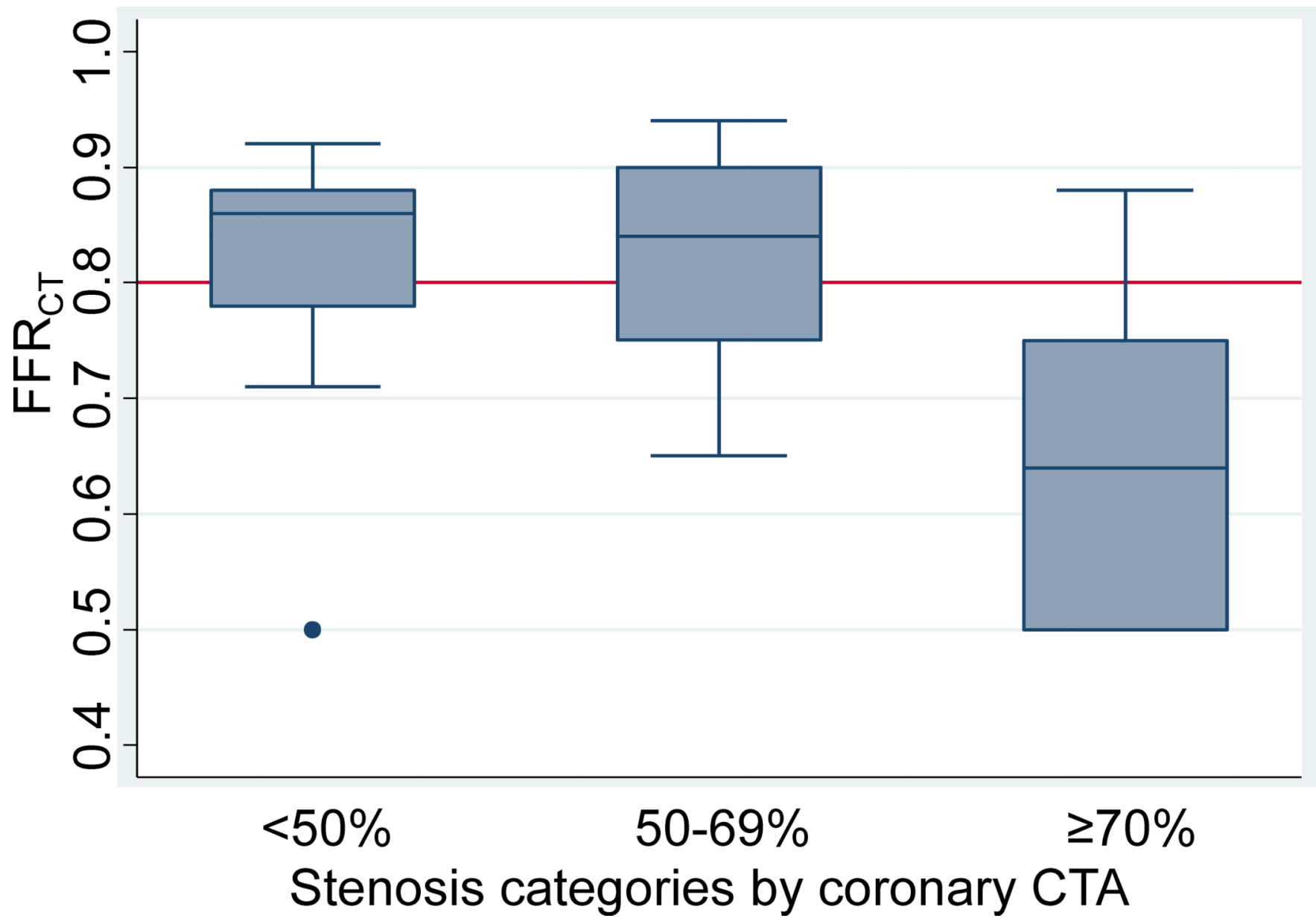

Figure 3.

Box and whisker plot of per patient $\mathrm{FFR}_{\mathrm{CT}}$ by categories of coronary CTA stenosis. 


\section{Table 1}

Baseline characteristics of 68 subjects included in the study stratified by FFR ${ }_{C T}$ results.

\begin{tabular}{lccc}
\hline & $\mathbf{F F R}_{\mathbf{C T}} \mathbf{\mathbf { 9 . 8 0 } \mathbf { n } = \mathbf { 4 0 }}$ & $\mathbf{F F R}_{\mathbf{C T}}>\mathbf{0 . 8 0} \mathbf{n}=\mathbf{2 8}$ & $\mathbf{p ~ v a l u e}$ \\
\hline Age - mean \pm SD, years & $57.1 \pm 7.2$ & $53.9 \pm 9.7$ & 0.154 \\
Female gender - no. (\%) & $6(15.0)$ & $14(50.0)$ & 0.003 \\
Cardiovascular Risk factors - no. (\%) & & & \\
$\quad$ Hypertension & $24(60.0)$ & $17(60.7)$ & 1.000 \\
Diabetes mellitus & $11(27.5)$ & $5(17.9)$ & 0.400 \\
Dyslipidemia & $24(60.0)$ & $18(64.3)$ & 0.803 \\
Family history of premature CAD & $8(20.0)$ & $11(39.3)$ & 0.103 \\
Former or current smoker & $28(70.0)$ & $14(50.0)$ & 0.129 \\
Obesity (BMI $\left.230 \mathrm{~kg} / \mathrm{m}^{2}\right)$ & $18(45.0)$ & $12(42.9)$ & 1.000 \\
Medications & & & \\
Aspirin & $13(32.5)$ & $8(28.6)$ & 0.794 \\
Beta-blockers & $9(22.5)$ & $9(32.1)$ & 0.413 \\
ACE inhibitors/ARB & $14(35.0)$ & $9(32.1)$ & 1.000 \\
Calcium channel blocker & $5(12.5)$ & $1(3.6)$ & 0.389 \\
Statins & $15(37.5)$ & $10(35.7)$ & 1.000 \\
\hline
\end{tabular}


Table 2

Coronary CTA findings (site reads), acute coronary syndrome during index hospitalization and additional cardiac tests stratified by $\mathrm{FFR}_{\mathrm{CT}}$ results.

\begin{tabular}{|c|c|c|c|}
\hline & $\mathrm{FFR}_{\mathrm{CT}} \mathbf{} \mathbf{0 . 8 0 \mathrm { n } = 4 0}$ & $\mathrm{FFR}_{\mathrm{CT}}>0.80 \mathrm{n}=28$ & p value \\
\hline \multicolumn{4}{|l|}{ Coronary CTA stenosis - no. (\%) } \\
\hline Indeterminate & $1(2.5)$ & $1(3.6)$ & 1.000 \\
\hline Mild $(<50 \%)$ & $5(12.5)$ & $10(35.7)$ & 0.036 \\
\hline Moderate (50-69\%) & $6(15.9)$ & $12(42.9)$ & 0.013 \\
\hline Severe ( $\geq 70 \%$ or $\geq 50 \%$ left main) & $28(70.0)$ & $5(17.9)$ & $<0.001$ \\
\hline Acute coronary syndrome - no. $(\%)$ & $23(57.5)$ & $4(14.3)$ & $<0.001$ \\
\hline Myocardial infarction & $3(7.5)$ & $0(0.0)$ & \\
\hline Unstable angina pectoris & $20(50.0)$ & $4(14.3)$ & \\
\hline Invasive coronary angiography - no. $(\%)$ & $21(52.5)$ & $9(32.1)$ & 0.137 \\
\hline Coronary revascularization - no. $(\%)$ & $15(37.5)$ & $3(10.7)$ & 0.024 \\
\hline Percutaneous coronary intervention & $12(30.0)$ & $3(10.7)$ & \\
\hline Coronary artery bypass & $3(7.5)$ & $0(0.0)$ & \\
\hline Non-invasive stress test - no. (\%) & $15(37.5)$ & $17(60.7)$ & 0.084 \\
\hline
\end{tabular}




\section{Table 3}

Results of core laboratory assessment for stenosis, qualitative high-risk plaque and quantitative plaque stratified by $\mathrm{FFR}_{\mathrm{CT}}$ results (per vessel analysis).

\begin{tabular}{|c|c|c|c|}
\hline & $\left.\mathrm{FFR}_{\mathrm{CT}} \mathbf{} \mathbf{}\right) .80 \mathrm{n}=71$ & $\mathrm{FFR}_{\mathrm{CT}}>0.80 \mathrm{n}=175$ & p value \\
\hline \multicolumn{4}{|l|}{ Stenosis - no. (\%) } \\
\hline Mild (<50\%) & $37(52.1)$ & $170(97.1)$ & $<0.001$ \\
\hline Moderate (50-69\%) & $13(18.3)$ & $4(2.3)$ & $<0.001$ \\
\hline Severe ( $\geq 70 \%$ or $\geq 50 \% \mathrm{LM}$ ) & $21(29.6)$ & $1(0.6)$ & $<0.001$ \\
\hline \multicolumn{4}{|l|}{ Qualitative plaque - no. (\%) } \\
\hline Any high-risk plaque & $48(67.6)$ & $52(29.7)$ & $<0.001$ \\
\hline Positive remodeling & $23(32.4)$ & $9(5.1)$ & $<0.001$ \\
\hline Low HU plaque & $15(23.1)$ & $5(2.9)$ & $<0.001$ \\
\hline Napkin-ring sign & $13(18.3)$ & $2(1.1)$ & $<0.001$ \\
\hline Spotty calcium & $41(57.8)$ & $50(28.6)$ & $<0.001$ \\
\hline \multicolumn{4}{|l|}{ Quantitative plaque - mean \pm SD } \\
\hline Total plaque volume, $\mathrm{mm}^{3}$ & $198.5 \pm 188.8$ & $43.2 \pm 79.7$ & $<0.001$ \\
\hline Plaque Volume $<30 \mathrm{HU}, \mathrm{mm}^{3}$ & $4.7 \pm 7.6$ & $1.1 \pm 4.7$ & $<0.001$ \\
\hline Remodeling index & $1.24 \pm 0.29$ & $1.05 \pm 0.14$ & $<0.001$ \\
\hline
\end{tabular}

